\title{
Higher harmonic analyses of the [252]Cf source driven noise analysis method
}

$\operatorname{AUTHOR}(S):$

Yamamoto, Toshihiro

CITATION:

Yamamoto, Toshihiro. Higher harmonic analyses of the [252]Cf source driven noise analysis method. Annals of Nuclear Energy 2015, 76: 521529

ISSUE DATE:

2015-02

URL:

http://hdl.handle.net/2433/193278

\section{RIGHT:}

C 2014 Elsevier Ltd.; この論文は出版社版でありません。引用の際には 出版社版をご確認ご利用ください。; This is not the published version. Please cite only the published version. 


\title{
Higher Harmonic Analyses of the ${ }^{252}$ Cf Source Driven Noise Analysis Method
}

\section{Toshihiro Yamamoto*}

Research Reactor Institute, Kyoto University, 2 Asashiro Nishi, Kumatori-cho, Sennan-gun, Osaka, 590-0494, Japan

\begin{abstract}
A theoretical formula has been derived to reconstruct the ratio of spectral densities in the ${ }^{252} \mathrm{Cf}$ source driven noise analysis (CSDNA) method from the higher harmonic eigenvalues and eigenfunctions of the $\alpha$-mode neutron transport equations. The formula closely reproduces each power spectral density and the ratio of spectral densities calculated by Monte Carlo simulations, thereby verifying the theoretical formula. The reactivity or $k_{e f f}$ of a subcritical system is related to the ratio of spectral densities by the fundamental mode approximation in which the higher harmonic modes are neglected. However, the ratio of spectral densities measured in the CSDNA method yields an ambiguous reactivity or $k_{\text {eff }}$ that depends on the locations of detectors due to the effect of the higher harmonics. A more elaborate method developed by Mihalczo and Valentine et al. infers an “experimental” $k_{\text {eff }}$ using a measurement and accurate Monte Carlo simulation of CSDNA. This paper discusses the uniqueness of the inferred “experimental” $k_{\text {eff }}$.
\end{abstract}

Keywords: noise analysis; ${ }^{252} \mathrm{Cf}$; higher harmonics; Monte Carlo; subcriticality

\footnotetext{
${ }^{*}$ Corresponding author. Tel:+81 72451 2414; fax:+81 724512658

E-mail address: toshihiro.yamamoto223@gmail.com (T.Yamamoto)
} 


\section{Introduction}

The ${ }^{252} \mathrm{Cf}$ source driven noise analysis (CSDNA) method (Paré and Mihalczo, 1975) has long been developed and investigated as a distinctive technique for the measurement of subcriticality. The CSDNA method uses an ionization chamber containing a ${ }^{252} \mathrm{Cf}$ source and provides the ratio of spectral densities, $R(\omega)$, that can be used for estimating the absolute value of the subcriticality. Unlike other familiar subcriticality measurement techniques, the CSDNA method is free from the requirements of measuring the detector efficiency, neutron source intensity, and reference subcriticality near criticality. A large number of papers have thus far been published dealing with subcriticality measurements of fissile materials or reactors utilizing the CSDNA method (e.g., Mihalczo et al., 1978, Mihalczo et al., 1990, Mihalczo et al., 1991, Mihalczo and Valentine, 1995, Hutchinson and Valentine, 2009, and Hutchinson and Bess, 2009). A Monte Carlo calculation method for simulating the CSDNA method was developed by Ficaro and Wehe (1994) and Valentine and Mihalczo (1996). The ratio of spectral densities in the CSDNA method is defined by

$$
R(\omega)=\frac{G_{12}^{*}(\omega) G_{13}(\omega)}{G_{11}(\omega) G_{23}(\omega)},
$$

where $G_{i j}(\omega)$ denotes an auto- or cross-power spectral density and the asterisk denotes complex conjugation, and $\omega$ is frequency. The subscript 1 refers to an ionization chamber containing a ${ }^{252} \mathrm{Cf}$ source, which triggers fission chain reactions within the subcritical multiplying system. The subscripts 2 and 3 refer to a pair of neutron detectors that detect the fission chain reactions initiated by spontaneous fission source neutrons from detector 1 . Actually spontaneous fission neutrons are emitted from fuel material, affecting the fission chain reactions in the subcritical system (Spriggs et al, 1999). However, this effect is neglected throughout this paper. There have been controversies among researchers over the interpretation of the ratio of spectral densities and how the ratio is related to the reactivity of a subcritical system (Yamane and Nishina, 1986, Difilippo, 1990, Sutton and Doub, 1991, Akcasu and 
Stolle, 1993). The measured quantities in subcritical measurements include the higher harmonic modes as well as the fundamental mode. Under the point-kinetics approximation (i.e., the fundamental mode approximation), the ratio $R$ is a real number and is independent of frequency and detectors' locations. In fact, the CSDNA method is affected by the higher harmonic modes. The ratio $R$ is a complex number and depends on frequency. The ratio $R$ definitely depends on the spatial arrangement of the three detectors. Under special circumstances where the subcritical system is nearly critical, the fundamental mode is predominant over the higher harmonic modes, and the relationship between the ratio $R$ and the reactivity is undoubtedly clear. Previous work by Yamane et al. (1987), Difilippo (1990), Sutton and Doub (1991), and Akcasu and Stolle (1993) considered the effects of the higher harmonic modes for the CSDNA method and derived theoretical formulae. However, the formulations have not yet been verified either experimentally or numerically.

In the present paper, a theoretical formula is derived for each spectral density in the ratio $R$ that considers the spatial and energy dependence. While a similar derivation has been carried out by Sutton and Doub (1991), the present paper poses slightly different expressions based on previous work on the higher harmonic effects on reactor noise theory (Endo et al., 2006; Yamamoto, 2011a; Yamamoto, 2014a; Yamamoto, 2014b). The expressions for the power spectral densities in these papers were derived mainly for the noise measurements in accelerator driven systems (ADS) in which spallation neutrons are emitted with a constant time interval. The expressions of the spectral densities were verified through comparison with Monte Carlo simulations for noise measurements in ADSs. Following the procedure in these papers on the reactor noise in ADSs, a rigorous formulation for the ratio $R$ can be made. Throughout this paper, delayed neutrons are neglected, and only prompt neutrons are considered. The ratio $R$ is to be theoretically reconstructed using the eigenfunctions and eigenvalues for the higher harmonics of the kinetics mode (i.e., $\alpha$-mode) transport equation. The reconstructed ratio $R$ is to be compared with the results of Monte Carlo simulations for the 
CSDNA method in very simplified numerical experiments. The relationship between the ratio $R$ and the reactivity (or $k_{e f f}$ ) is derived by introducing the fundamental mode approximation to the CSDNA method. We discuss how the $k_{\text {eff }}$ obtained from the ratio $R$ depends on the spatial arrangement of the detectors. We review another approach by Mihalczo et al. (1997) and Valentine (1999) to infer $k_{\text {eff }}$ from the ratio $R$; it utilizes accurate Monte Carlo simulations of the CSDNA method. We consider this approach from a viewpoint of the uniqueness of the inferred "experimental” $k_{e f f}$.

\section{Formulations of the Ratio of Power Spectral Densities}

\subsection{Auto-power spectral density of an ionization chamber}

We derive here the auto-power spectral density (APSD), $G_{11}(\omega)$, of an ionization chamber containing a ${ }^{252} \mathrm{Cf}$ source . It is assumed that the alpha-decay of the ${ }^{252} \mathrm{Cf}$ source does not contribute to the detection of the chamber and that all detections are due to the spontaneous fissions. This can be achieved by setting an appropriate discriminator threshold. The responses of the electronic components of the detection system, denoted by $h_{1}(\omega), h_{2}(\omega)$, and $h_{3}(\omega)$ in (Mihalczo et al., 1990), are not considered in this paper because they are all eventually eliminated in the ratio of spectral densities (Eq. (1)) (Yamane et al, 1986). The auto-correlation function of detector 1 for a time lag between two detection times, (i.e., $\tau=t_{2}-t_{1}$ ) is given by

$$
R_{11}(\tau) d t_{1} d t_{2}=\varepsilon_{1} S_{\text {int }} d t_{1} \cdot \varepsilon_{1} S_{\text {int }} d t_{2}+\varepsilon_{1} S_{\text {int }} \delta(\tau) d t_{1} d t_{2},
$$

where $\varepsilon_{1}$ = detection efficiency of detector 1 per spontaneous fission of ${ }^{252} \mathrm{Cf}$, and $S_{\text {int }}=$ number of fissions per unit time in the ${ }^{252} \mathrm{Cf}$ source. Given that the ${ }^{252} \mathrm{Cf}$ source intensity per unit volume has a spatial distribution $S(\mathbf{r}), S_{\text {int }}$ is given by

$$
S_{\text {int }}=\int_{V} S(\mathbf{r}) d \mathbf{r} \text {. }
$$

Fourier transforming $R_{11}(\tau)$ in Eq. (2) yields the APSD of detector 1:

$$
G_{11}(\omega)=\varepsilon_{1} S_{\text {int }} \text {, for } \omega \neq 0 \text {. }
$$




\subsection{Cross-power spectral density between detectors 2 and 3}

To derive the formulation of the cross-power spectral density (CPSD) between detectors 2 and 3, we start with the derivation of the cross-correlation function between the two detectors. The correlation between the detections in the two detectors stems from the fact that two or more neutrons are emitted simultaneously from a spontaneous fission of ${ }^{252} \mathrm{Cf}$ or a neutron-induced fission. The formulation for the CPSD has been derived in previous papers (Sutton and Doub, 1991; Endo et al, 2006; Yamamoto, 2014a; Yamamoto, 2014b), but is presented again here.

First, a Green's function that represents the neutron density at $(\mathbf{r}, E, \boldsymbol{\Omega}, t)$ due to one neutron emitted at $\left(\mathbf{r}_{0}, E_{0}, \mathbf{\Omega}_{0}, t_{0}\right)$ is introduced (Sutton and Doub, 1991; Endo et al., 2006):

$$
\begin{aligned}
& G\left(\mathbf{r}, E, \boldsymbol{\Omega}, t \mid \mathbf{r}_{0}, E_{0}, \mathbf{\Omega}_{0}, t_{0}\right)=G\left(\mathbf{r}, E, \boldsymbol{\Omega} \mid \mathbf{r}_{0}, E_{0}, \mathbf{\Omega}_{0} ; t-t_{0}\right) \\
& =\frac{1}{v(E)} \sum_{\ell=0}^{\infty} \psi_{\ell}(\mathbf{r}, E, \boldsymbol{\Omega}) \psi_{\ell}^{*}\left(\mathbf{r}_{0}, E_{0}, \mathbf{\Omega}_{0}\right) e^{-\alpha_{\ell}\left(t-t_{0}\right)} .
\end{aligned}
$$

This Green's function is slightly different from the original expression in that the right-hand side is divided by the neutron velocity $v(E) . \psi_{\ell}(\mathbf{r}, E, \boldsymbol{\Omega})$ and $\psi_{\ell}^{*}(\mathbf{r}, E, \boldsymbol{\Omega})$ are the $\ell$ th eigenfunctions of the forward and adjoint $\alpha$-mode eigenvalue equations at position $\mathbf{r}$ with energy $E$ and direction $\boldsymbol{\Omega}$, respectively:

$$
\begin{aligned}
& -\boldsymbol{\Omega} \cdot \nabla \psi_{\ell}(\mathbf{r}, E, \boldsymbol{\Omega})-\Sigma_{t}(\mathbf{r}, E) \psi_{\ell}(\mathbf{r}, E, \boldsymbol{\Omega}) \\
& +\int_{4 \pi} d \mathbf{\Omega}^{\prime} \int d E^{\prime} \Sigma_{s}\left(\mathbf{r}, \boldsymbol{\Omega}^{\prime} \rightarrow \boldsymbol{\Omega}, E^{\prime} \rightarrow E\right) \psi_{\ell}\left(\mathbf{r}, E^{\prime}, \mathbf{\Omega}^{\prime}\right) \\
& +\frac{\chi_{f}(E)}{4 \pi} \int_{4 \pi} d \boldsymbol{\Omega}^{\prime} \int d E^{\prime} v \Sigma_{f}\left(\mathbf{r}, E^{\prime}\right) \psi_{\ell}\left(\mathbf{r}, E^{\prime}, \boldsymbol{\Omega}^{\prime}\right)+\frac{\alpha_{\ell}}{v(E)} \psi_{\ell}(\mathbf{r}, E, \boldsymbol{\Omega})=0, \\
& \mathbf{\Omega} \cdot \nabla \psi_{\ell}^{*}(\mathbf{r}, E, \boldsymbol{\Omega})-\Sigma_{t}(\mathbf{r}, E) \psi_{\ell}^{*}(\mathbf{r}, E, \boldsymbol{\Omega}) \\
& +\int_{4 \pi} d \boldsymbol{\Omega}^{\prime} \int d E^{\prime} \Sigma_{s}\left(\mathbf{r}, \boldsymbol{\Omega} \rightarrow \boldsymbol{\Omega}^{\prime}, E \rightarrow E^{\prime}\right) \psi_{\ell}^{*}\left(\mathbf{r}, E^{\prime}, \boldsymbol{\Omega}^{\prime}\right) \\
& +\frac{v \Sigma_{f}(\mathbf{r}, E)}{4 \pi} \int_{4 \pi} d \boldsymbol{\Omega}^{\prime} \int d E^{\prime} \chi_{f}\left(E^{\prime}\right) \psi_{\ell}^{*}\left(\mathbf{r}, E^{\prime}, \mathbf{\Omega}^{\prime}\right)+\frac{\alpha_{\ell}}{v(E)} \psi_{\ell}^{*}(\mathbf{r}, E, \boldsymbol{\Omega})=0,
\end{aligned}
$$

where $\Sigma_{t}=$ the macroscopic total cross section, $\Sigma_{s}=$ the macroscopic scattering cross section, $\Sigma_{f}=$ the macroscopic fission cross section, $\chi_{f}(E)=$ the prompt neutron spectrum, and $v=$ the number of prompt neutrons per fission. The adjoint and forward eigenfunctions are 
normalized as

$$
\int_{V} d \mathbf{r} \int_{0}^{\infty} d E \int_{4 \pi} d \mathbf{\Omega} \frac{1}{v(E)} \psi_{m}^{*}(\mathbf{r}, E, \boldsymbol{\Omega}) \psi_{n}(\mathbf{r}, E, \boldsymbol{\Omega})=\delta_{m n},
$$

where $\delta_{m n}$ is the Kronecker delta. Using the Green's function of Eq. (5), the fission reaction rate at $\left(\mathbf{r}, t_{f}\right)$ caused by $\bar{q}$ neutrons emitted from a ${ }^{252} \mathrm{Cf}$ spontaneous fission at $t_{0}$ is given by

$$
\begin{aligned}
& F\left(\mathbf{r}, t_{f} ; t_{0}\right) \equiv \int_{0}^{\infty} d E \int_{4 \pi} d \boldsymbol{\Omega} \int_{V} d \mathbf{r}_{0} \int_{0}^{\infty} d E_{0} \int_{4 \pi} d \mathbf{\Omega}_{0} v(E) \Sigma_{f}(\mathbf{r}, E) \\
& \times G\left(\mathbf{r}, E, \boldsymbol{\Omega} \mid \mathbf{r}_{0}, E_{0}, \boldsymbol{\Omega}_{0} ; t_{f}-t_{0}\right) \bar{q} S\left(\mathbf{r}_{0}\right) \chi_{s}\left(E_{0}, \mathbf{\Omega}_{0}\right) \\
& =\sum_{\ell=0}^{\infty} \int_{0}^{\infty} d E \int_{4 \pi} d \boldsymbol{\Omega} \Sigma_{f}(\mathbf{r}, E) \psi_{\ell}(\mathbf{r}, E, \boldsymbol{\Omega}) S_{\ell} e^{-\alpha_{\ell}\left(t_{f}-t_{0}\right)},
\end{aligned}
$$

where $\chi_{s}\left(E_{0}, \Omega_{0}\right)=$ the energy and direction distributions of fission neutrons from the ${ }^{252} \mathrm{Cf}$ source, and $S_{\ell}$ is defined by

$$
S_{\ell}=\bar{q} \int_{V} d \mathbf{r}_{0} \int_{0}^{\infty} d E_{0} \int_{4 \pi} d \boldsymbol{\Omega}_{0} S\left(\mathbf{r}_{0}\right) \chi_{s}\left(E_{0}, \boldsymbol{\Omega}_{0}\right) \psi_{\ell}^{*}\left(\mathbf{r}_{0}, E_{0}, \mathbf{\Omega}_{0}\right)
$$

The cross-correlation function of a pair of neutron detections at $t_{1}$ by detector 2 and at $t_{2}$ $\left(t_{2}>t_{1}\right)$ by detector 3 resulting from the fission at $t_{f}$ is

$$
\begin{aligned}
& R_{f}\left(\tau, t_{f}\right)=\sum_{v=0}^{\infty} p_{f}(v) \int_{-\infty}^{t_{f}} d t_{0} \int_{V} d \mathbf{r}_{0} \int_{0}^{\infty} d E_{0} \int_{4 \pi} d \mathbf{\Omega}_{0} F\left(\mathbf{r}_{0}, t_{f} ; t_{0}\right) \\
& \times \int_{V} d \mathbf{r} \int_{0}^{\infty} d E \int_{4 \pi} d \boldsymbol{\Omega} v(E) \Sigma_{d 2}(\mathbf{r}, E) G\left(\mathbf{r}, E, \boldsymbol{\Omega} \mid \mathbf{r}_{0}, E_{0}, \boldsymbol{\Omega}_{0} ; t_{1}-t_{f}\right) v \frac{\chi_{f}\left(E_{0}\right)}{4 \pi} \\
& \times \int_{V} d \mathbf{r} \int_{0}^{\infty} d E \int_{4 \pi} d \mathbf{\Omega} v(E) \Sigma_{d 3}(\mathbf{r}, E) G\left(\mathbf{r}, E, \boldsymbol{\Omega} \mid \mathbf{r}_{0}, E_{0}, \mathbf{\Omega}_{0} ; t_{2}-t_{f}\right)(v-1) \frac{\chi_{f}\left(E_{0}\right)}{4 \pi} \\
& =\sum_{\ell=0}^{\infty} \sum_{m=0}^{\infty} \sum_{n=0}^{\infty} \frac{S_{\ell}}{\alpha_{\ell}} F_{\ell \rightarrow m n} D_{2 m} D_{3 n} e^{\left(\alpha_{m}+\alpha_{n}\right) t} t_{f} e^{-\alpha_{m} t_{1}} e^{-\alpha_{n} t_{2}},
\end{aligned}
$$

where $\Sigma_{d 2}=$ the cross section of detector 2 and $p_{f}(v)=$ the probability density function of the number of induced fission neutrons. $F_{\ell \rightarrow m n}$ and $D_{2 m}$ are defined as follows:

$$
\begin{gathered}
F_{\ell \rightarrow m n}=\overline{v(v-1)} \int_{V} d \mathbf{r} \int_{0}^{\infty} d E \int_{4 \pi} d \mathbf{\Omega} \Sigma_{f}(\mathbf{r}, E) \psi_{\ell}(\mathbf{r}, E, \boldsymbol{\Omega}) I_{f, m}(\mathbf{r}) I_{f, n}(\mathbf{r}), \\
I_{f, m}(\mathbf{r})=\int_{0}^{\infty} d E \int_{4 \pi} d \boldsymbol{\Omega} \frac{\chi_{f}(E)}{4 \pi} \psi_{m}^{*}(\mathbf{r}, E, \boldsymbol{\Omega}) \\
D_{2 m}=\int_{V} d \mathbf{r} \int_{0}^{\infty} d E \int_{4 \pi} d \mathbf{\Omega} \Sigma_{d 2}(\mathbf{r}, E) \psi_{m}(\mathbf{r}, E, \boldsymbol{\Omega})
\end{gathered}
$$

The cross-correlation function of a pair of neutron detections at $t_{1}$ by detector 2 and at $t_{2}$ 
$\left(t_{2}>t_{1}, \quad \tau=t_{2}-t_{1}\right)$ by detector 3 resulting from all fissions before $t_{1}$ is

$$
R_{f}(\tau)=\int_{-\infty}^{t_{1}} R_{f}\left(\tau, t_{f}\right) d t_{f}=\sum_{\ell=0}^{\infty} \sum_{m=0}^{\infty} \sum_{n=0}^{\infty} \frac{S_{\ell} F_{\ell \rightarrow m n} D_{2 m} D_{3 n}}{\alpha_{\ell}\left(\alpha_{m}+\alpha_{n}\right)} e^{-\alpha_{n} \tau} .
$$

Next, the cross-correlation between the detectors 2 and 3 due to multiple neutron emission from the ${ }^{252} \mathrm{Cf}$ source is considered. The cross-correlation function of a pair of neutron detections at $t_{1}$ by detector 2 and at $t_{2} \quad\left(t_{2}>t_{1}\right)$ by detector 3 resulting from all spontaneous fissions before $t_{1}$ is

$$
\begin{aligned}
& R_{S}(\tau)=\sum_{q=0}^{\infty} p_{s}(q) \int_{-\infty}^{t_{1}} d t_{0} \int_{V} d \mathbf{r}_{0} \int_{0}^{\infty} d E_{0} \int_{4 \pi} d \mathbf{\Omega}_{0} S\left(\mathbf{r}_{0}\right) \\
& \times \int_{V} d \mathbf{r} \int_{0}^{\infty} d E \int_{4 \pi} d \boldsymbol{\Omega} v(E) \Sigma_{d 2}(\mathbf{r}, E) G\left(\mathbf{r}, E, \boldsymbol{\Omega} \mid \mathbf{r}_{0}, E_{0}, \mathbf{\Omega}_{0} ; t_{1}-t_{0}\right) q \chi_{s}\left(E_{0}, \mathbf{\Omega}_{0}\right) \\
& \times \int_{V} d \mathbf{r} \int_{0}^{\infty} d E \int_{4 \pi} d \boldsymbol{\Omega} v(E) \Sigma_{d 3}(\mathbf{r}, E) G\left(\mathbf{r}, E, \boldsymbol{\Omega} \mid \mathbf{r}_{0}, E_{0}, \mathbf{\Omega}_{0} ; t_{2}-t_{0}\right)(q-1) \chi_{s}\left(E_{0}, \mathbf{\Omega}_{0}\right) \\
& =\sum_{m=0}^{\infty} \sum_{n=0}^{\infty} S_{m n} \frac{D_{2 m} D_{3 n}}{\alpha_{m}+\alpha_{n}} e^{-\alpha_{n} \tau}
\end{aligned}
$$

Where $p_{s}(q)=$ the probability density function of the number of spontaneous fission neutrons,

$$
\begin{aligned}
& S_{m n}=\overline{q(q-1)} \int_{V} d \mathbf{r}_{0} S\left(\mathbf{r}_{0}\right) I_{s, m}\left(\mathbf{r}_{0}\right) I_{s, n}\left(\mathbf{r}_{0}\right), \\
& I_{s, m}\left(\mathbf{r}_{0}\right)=\int_{0}^{\infty} d E_{0} \int_{4 \pi} d \mathbf{\Omega}_{0} \chi_{s}\left(E_{0}, \mathbf{\Omega}_{0}\right) \psi_{m}^{*}\left(\mathbf{r}_{0}, E_{0}, \mathbf{\Omega}_{0}\right) .
\end{aligned}
$$

The CPSD between detectors 2 and $3, G_{23}(\omega)$, is obtained as the Fourier transform of the cross-correlation functions $R_{f}(\tau)$ and $R_{s}(\tau)$ :

$$
\begin{aligned}
& G_{23}(\omega)=\int_{-\infty}^{\infty}\left(R_{f}(\tau)+R_{s}(\tau)\right) e^{-i \omega \tau} d \tau \\
& =\sum_{m=0}^{\infty} \sum_{n=0}^{\infty} A_{m 2, n 3} \int_{0}^{\infty} e^{-\alpha_{n} \tau} e^{-i \omega \tau} d \tau+\sum_{m=0}^{\infty} \sum_{n=0}^{\infty} A_{m 3, n 2} \int_{-\infty}^{0} e^{\alpha_{n} \tau} e^{-i \omega \tau} d \tau \\
& =\sum_{m=0}^{\infty} \sum_{n=0}^{\infty}\left(A_{m 2, n 3} \frac{1}{\alpha_{n}+i \omega}+A_{m 3, n 2} \frac{1}{\alpha_{n}-i \omega}\right),
\end{aligned}
$$

where $i=\sqrt{-1}$, and

$$
A_{m 2, n 3}=\left(\sum_{\ell=0}^{\infty}\left(\frac{S_{\ell} F_{\ell \rightarrow m n}}{\alpha_{\ell}}\right)+S_{m n}\right) \frac{D_{2 m} D_{3 n}}{\left(\alpha_{m}+\alpha_{n}\right)}
$$




\subsection{Cross-power spectral density between detectors 1 and 2 or 3}

Following the derivations for the APSD and the CPSD presented thus far, the cross-correlation function of a pair of neutron detections at $t_{0}$ by detector 1 and at $t_{1}\left(t_{1}>t_{0}\right.$, $\left.\tau=t_{1}-t_{0}\right)$ by detector 2 is given by

$$
\begin{aligned}
& R_{12}(\tau)=\int_{-\infty}^{t_{1}} d t_{0} \int_{V} d \mathbf{r}_{0} \int_{0}^{\infty} d E_{0} \int_{4 \pi} d \mathbf{\Omega}_{0} \varepsilon_{1} \bar{q} S\left(\mathbf{r}_{0}\right) \chi_{s}\left(E_{0}, \mathbf{\Omega}_{0}\right) \\
& \times \int_{V} d \mathbf{r} \int_{0}^{\infty} d E \int_{4 \pi} d \mathbf{\Omega} v(E) \Sigma_{d 2}(\mathbf{r}, E) G\left(\mathbf{r}, E, \boldsymbol{\Omega} \mid \mathbf{r}_{0}, E_{0}, \mathbf{\Omega}_{0} ; t_{1}-t_{0}\right) \\
& =\varepsilon_{1} \sum_{m=0}^{\infty} S_{m} D_{2 m} e^{-\alpha_{m} \tau}
\end{aligned}
$$

Neutron detections in detector 2 or 3 always follow a spontaneous fission in detector 1 . Thus, noting that the integration range of the Fourier transformation of Eq. (21) is from 0 to $\infty$, we obtain the CPSD between detectors 1 and 2:

$$
G_{12}(\omega)=\int_{0}^{\infty} R_{12}(\tau) e^{-i \omega \tau} d \tau=\varepsilon_{1} \sum_{m=0}^{\infty} \frac{S_{m} D_{2 m}}{\alpha_{m}+i \omega} .
$$

\subsection{Ratio of spectral densities and fundamental mode approximation}

As a result, we obtain the ratio of spectral densities of the CSDNA method that takes into account the spatial and energy dependence:

$$
R(\omega)=\frac{G_{12}^{*}(\omega) G_{13}(\omega)}{G_{11}(\omega) G_{23}(\omega)}=\frac{\varepsilon_{1} \sum_{m=0}^{\infty} \sum_{n=0}^{\infty} S_{m} S_{n} D_{2 m} D_{3 n} \frac{1}{\alpha_{m}-i \omega} \cdot \frac{1}{\alpha_{n}+i \omega}}{S_{\text {int }} \sum_{m=0}^{\infty} \sum_{n=0}^{\infty}\left(A_{m 2, n 3} \frac{1}{\alpha_{n}+i \omega}+A_{m 3, n 2} \frac{1}{\alpha_{n}-i \omega}\right)} .
$$

The ratio of spectral densities, $R(\omega)$, is in general a complex number and is not independent of frequency. If the higher harmonic components are neglected except for $\ell=m=n=0$, the ratio of spectral densities becomes

$$
R_{0} \equiv \frac{\varepsilon_{1} S_{0}^{2}}{S_{\text {int }}\left(\frac{S_{0} F_{0 \rightarrow 00}}{\alpha_{0}}+S_{00}\right)} .
$$

Thus, by introducing the fundamental mode approximation, the ratio of spectral densities becomes a real number and independent of frequency. 
Next, consider how to relate $R_{0}$ to the reactivity or $k_{\text {eff. }}$ In the absence of delayed neutrons, the reactivity $\rho\left(=1-1 / k_{\text {eff }}\right)$ is given by the product of the fundamental mode $\alpha_{0}$ and the (prompt) neutron generation time $\Lambda$ :

$$
\rho=-\alpha_{0} \Lambda
$$

The (prompt) neutron generation time, $\Lambda$, is given by

$$
\Lambda=\frac{\left\langle\phi_{0}^{*}(\mathbf{r}, E, \mathbf{\Omega}) \frac{1}{v(E)} \psi_{0}(\mathbf{r}, E, \mathbf{\Omega})\right\rangle}{\left\langle I_{\lambda f, m}(\mathbf{r}) v \Sigma_{f}(\mathbf{r}, E) \psi_{0}(\mathbf{r}, E, \mathbf{\Omega})\right\rangle},
$$

where the angle brackets denote integration over all phase space, and $\phi_{0}^{*}=$ the fundamental mode adjoint flux of the $\lambda$-mode transport eigenvalue equation:

$$
\begin{aligned}
& \mathbf{\Omega} \cdot \nabla \phi_{0}^{*}(\mathbf{r}, E, \boldsymbol{\Omega})-\Sigma_{t}(\mathbf{r}, E) \phi_{0}^{*}(\mathbf{r}, E, \boldsymbol{\Omega}) \\
& +\int_{4 \pi} d \mathbf{\Omega}^{\prime} \int_{0}^{\infty} d E^{\prime} \Sigma_{s}\left(\mathbf{r}, \boldsymbol{\Omega} \rightarrow \boldsymbol{\Omega}^{\prime}, E \rightarrow E^{\prime}\right) \phi_{0}^{*}\left(\mathbf{r}, E^{\prime}, \mathbf{\Omega}^{\prime}\right) \\
& +\frac{v \Sigma_{f}(\mathbf{r}, E)}{4 \pi k_{e f f}} \int_{4 \pi} d \mathbf{\Omega}^{\prime} \int_{0}^{\infty} d E^{\prime} \chi_{f}\left(E^{\prime}\right) \phi_{0}^{*}\left(\mathbf{r}, E^{\prime}, \mathbf{\Omega}^{\prime}\right)=0,
\end{aligned}
$$

and

$$
I_{\lambda f, m}(\mathbf{r})=\int_{0}^{\infty} d E \int_{4 \pi} d \mathbf{\Omega} \frac{\chi_{f}(E)}{4 \pi} \phi_{0}^{*}(\mathbf{r}, E, \mathbf{\Omega}) .
$$

Here, we assume that $\phi_{0}^{*} \approx \psi_{0}^{*}$ and $I_{\lambda f, m}(\mathbf{r}) \approx I_{f, m}(\mathbf{r})$. Then, using the orthonormality relationship in Eq. (8) for the numerator of Eq. (26), we obtain

$$
\Lambda \approx \frac{1}{\left\langle I_{f, 0}(\mathbf{r}) v \Sigma_{f}(\mathbf{r}, E) \psi_{0}(\mathbf{r}, E, \mathbf{\Omega})\right\rangle} .
$$

Using Eqs. (24), (25), and (29), we find a relationship between the reactivity $\rho$ and $R_{0}$ :

$$
R_{0} \equiv \frac{\varepsilon_{1} S_{0}^{2}}{S_{\text {int }}\left(-\frac{S_{0} F_{0 \rightarrow 00}}{\rho\left\langle I_{f, 0}(\mathbf{r}) v \Sigma_{f}(\mathbf{r}, E) \psi_{0}(\mathbf{r}, E, \boldsymbol{\Omega})\right\rangle}+S_{00}\right)},
$$

where

$$
\begin{aligned}
& S_{0}=\bar{q}\left\langle S(\mathbf{r}) \chi_{s}(E, \mathbf{\Omega}) \psi_{0}^{*}(\mathbf{r}, E, \mathbf{\Omega})\right\rangle, \\
& F_{0 \rightarrow 00}=\overline{v(v-1)}\left\langle\Sigma_{f}(\mathbf{r}, E) \psi_{0}(\mathbf{r}, E, \mathbf{\Omega}) I_{f, 0}(\mathbf{r}) I_{f, 0}(\mathbf{r})\right\rangle, \\
& S_{00}=\overline{q(q-1)}\left\langle S(\mathbf{r}) I_{s, 0}(\mathbf{r}) I_{s, 0}(\mathbf{r})\right\rangle .
\end{aligned}
$$

The constants $S_{0}, F_{0 \rightarrow 00}$, and $S_{00}$ used in Eq. (30) are obtained by calculations. While $R_{0}$ by the fundamental mode approximation does not depend on the locations of detectors 2 and 3 , it depends on the location of detector 1, i.e., $S(\mathbf{r})$. When we furthermore neglect the spatial 
and energy dependence (i.e., point-kinetics approximation), $R_{0}$ in Eq. (30) becomes

$$
R_{0, p} \equiv \frac{\varepsilon_{1} \bar{q}^{2}}{-\frac{\bar{q}}{\rho} \overline{\frac{v(v-1)}{\bar{v}}}+\overline{q(q-1)}},
$$

where the subscript $p$ denotes the point-kinetics approximation. While Sutton and Doub (1991) related the reactivity to $R(\omega)$ without introducing the fundamental mode approximation, the present paper does so by neglecting all higher harmonic components except for the fundamental mode. As a subcritical multiplying system becomes critical, $\alpha_{0}$ approaches zero in the absence of delayed neutrons and $\alpha_{0}<<\alpha_{1}<\alpha_{2}<\cdots$. As one can see in Eqs. (19), (20) and (22), $\alpha_{m}$ and $\alpha_{n}$ are in the denominators of $G_{12}(\omega), G_{13}(\omega)$, and $G_{23}(\omega)$. Thus when $\alpha_{0}$ is very close to zero, the terms including $\alpha_{m}$ and $\alpha_{n}(m, n \geq 1)$ in $G_{12}(\omega), G_{13}(\omega)$, and $G_{23}(\omega)$ become negligibly small compared with the terms of the fundamental mode. Therefore, the fundamental mode approximation in Eq. (30) can be more accurate for a subcritical system that is nearly critical.

\section{Numerical Tests}

\subsection{Description of the numerical test}

We have applied the rigorous formula of the ratio of spectral densities to a subcritical multiplying system whose geometry is a one-dimensional infinite slab. This example is the same as that in the author's previous paper (Yamamoto, 2014b). The energy-dependence is neglected in the numerical tests in this paper. As shown in Fig. 1, the slab has a thickness of 55 cm. Vacuum boundary conditions are imposed on both sides of the slab. The multiplying system has the following properties:

$$
\begin{gathered}
\Sigma_{t}=0.28 \mathrm{~cm}^{-1}, \Sigma_{f}=0.049 \mathrm{~cm}^{-1}, \Sigma_{c}=0.05 \mathrm{~cm}^{-1}, v=2,200 \mathrm{~m} / \mathrm{s}, v=2, \\
k_{e f f}=0.95865 \pm 0.00002, q=3 .
\end{gathered}
$$

[Fig. 1]

The number of neutrons emitted by a neutron-induced fission and a spontaneous fission is 
assumed fixed. Thus, we set $\overline{v(v-1)}=v(v-1)=2$ and $\overline{q(q-1)}=q(q-1)=6$. Neutron scattering is assumed to be isotropic in the laboratory system. The eigenvalue calculations for $k_{\text {eff }}$ and $\alpha_{m}$ of this subcritical system were conducted using a test Monte Carlo program developed by the author. All Monte Carlo eigenvalue calculations, including the $\alpha$-mode calculations, were conducted with 40,000 neutrons per cycle, 100 discard cycles and 12,000 active cycles. To calculate the ratio of spectral densities as described in Eq. (23), a large number of higher order mode $\alpha$-eigenvalues and eigenfunctions (flux distributions) are needed. A Monte Carlo calculation method developed by Yamamoto (2011b) for solving higher harmonics $\alpha$-mode eigenvalue problems was used up to the third order harmonics. In general, lower order harmonics are more important for mode analyses of a subcritical system. The accurate results based on neutron transport theory were used up to the third order harmonics. Beyond the third order, the $\alpha$-eigenvalues and flux distributions were approximated with diffusion theory:

$$
\begin{aligned}
\alpha_{m} & =v\left(\Sigma_{f}+\Sigma_{c}+D B_{m}^{2}-v \Sigma_{f}\right), \quad m \geq 4, \\
\psi_{m}(x) & =\psi_{m}^{*}(x)=\sqrt{\frac{2 v}{H+2 d}} \sin B_{m}(x+d), \quad 0 \leq x \leq H, m \geq 4,
\end{aligned}
$$

where

$$
B_{m}=\frac{(m+1) \pi}{H+2 d},
$$

$D=$ diffusion coefficient $\left(=1 / 3 \Sigma_{t}\right), H=$ thickness of the slab, and $d=$ extrapolated length $\left(=0.7104 / \Sigma_{t}\right)$. The orthonormality condition is

$$
\frac{1}{v} \int_{-d}^{H+d} \psi_{m}(x) \psi_{n}^{*}(x) d x=\delta_{m n} .
$$

Table 1 compares the $\alpha$-eigenvalues calculated with the Monte Carlo method with the diffusion theory. These results are reproduced from (Yamamoto, 2014b). The diffusion theory agrees well with the transport theory for the fundamental mode. As the order increases, the difference between two methods increases. Because the energy-dependence is not considered in 
this paper, the angular adjoint flux for $0 \leq m \leq 3$ is obtained by reversing the angular distribution of the forward flux: $\psi_{m}^{*}(\mathbf{r}, \mathbf{\Omega})=\psi_{m}(\mathbf{r},-\mathbf{\Omega})$.

The positions of the detectors and the ${ }^{252} \mathrm{Cf}$ source for the numerical test are shown in Fig. 1. The position of the ${ }^{252} \mathrm{Cf}$ source (detector 1$)(x=34.0 \mathrm{~cm})$ is off-center of the slab, which activates the asymmetric higher harmonic modes as well as the symmetric modes. Detectors 2 $\left(D_{2}\right)(x=41.4 \mathrm{~cm})$ and $3\left(D_{3}\right)(x=13.6 \mathrm{~cm})$ are located near the top and bottom of the first higher harmonic mode, respectively. The spectral densities defined by Eqs. (22) and (19) were calculated using the eigenvalues and the eigenfunctions of modes up to order 250. No higher-order modes substantially affect the spectral densities, as shown later.

[Table 1]

\subsection{Monte Carlo simulation method of the CSDNA method}

The spectral densities, $G_{11}(\omega), G_{12}(\omega), G_{13}(\omega)$, and $G_{23}(\omega)$ were obtained by performing a Monte Carlo simulation of the CSDNA method as the reference solutions. The calculation flow of the simulation is almost the same as in (Yamamoto, 2014a). The Monte Carlo simulation is based on the analog Monte Carlo technique in which any variance-reduction techniques are disabled. The calculation flow of the Monte Carlo simulation is as follows.

(1) A neutron from a spontaneous fission is emitted isotropically at $t=t_{0}$, which coincides with the starting time of the data blocks of the three detectors. A data block is made up of time samples of a detector response composed of $M=2^{13}=8192$ time bins. Each time bin has a width of $\Delta\left(=4 \times 10^{-5} \mathrm{~s}\right)$ and contains the number of detections that occur during the corresponding time bin. A spontaneous fission is registered in the time bin of detector 1 corresponding to the time $t$. This Monte Carlo simulation assumed that all spontaneous fissions were registered to detector 1 without count loss; thus, $\varepsilon_{1}=1$.

(2) The neutron flies to the next collision site. The flight distance $s$ is determined by $s=-\ln \xi_{1} / \Sigma_{t}$ where $\xi_{1}$ is a uniform pseudo-random number from $(0,1]$. The time of the 
neutron is updated to $t^{\prime}=t-s / v$.

(3) If $\xi_{2}<\Sigma_{c} / \Sigma_{t}$, the neutron is captured and the random walk terminates. When the capture occurs within the region of detector 2 or 3, one neutron count is registered in the respective time bin corresponding to time $t^{\prime}$. Each detector region is a thin layer with a width of $0.534 \mathrm{~cm}$. The width of the detector is the slab thickness $(55 \mathrm{~cm})$ divided by 103 .

(4) If the reaction is not a capture and $\xi_{3}<\Sigma_{f} / \Sigma_{t}$, the neutron induces a fission reaction and the random walk terminates. $v(=2)$ neutrons are stored in the fission source bank.

(5) If the reaction is a scattering, then determine the scattered neutron direction and return to step (2).

(6) If the neutron is killed by the capture or fission reaction but the fission source bank is not empty, a neutron is removed from the bank and emitted isotropically from the fission site. Then go to step (2). If the bank was empty, go to the next step.

(7) Steps (1) through (6) are repeated $q$ (= the number of neutrons emitted from the spontaneous fission) times.

(8) The time of the next spontaneous fission is updated to $t_{0}=t_{0}-\ell n \xi_{4} / S_{\text {int }}$, where $S_{\text {int }}$ is the number of spontaneous fissions of the ${ }^{252} \mathrm{Cf}$ source per unit time. In this simulation, $S_{\text {int }}=$ $4000 \mathrm{~s}^{-1}$. Then return to step (1). If $t_{0}$ exceeds the end of the data block, then calculate the correlation functions, APSD, and CPSDs using the data blocks. Clear the data blocks before returning to step (1).

(9) Steps (1) through (8) are continued until the desired statistics are obtained.

For calculating the APSD and the CPSDs from the data blocks, we used a data processing method employed in MCNP-DSP (Valentine and Mihalczo, 1996; Valentine and Mihalczo, 1997). The method to calculate the CPSDs is illustrated below. The circular cross-correlation function is obtained as: 


$$
R_{x y}(n)=\sum_{k=0}^{M-1} x_{k} y_{(M-(n-k))},
$$

where $x$ and $y$ are the detector signals of detectors 2 and 3, respectively, and $n=$ lag index. The detector signals are assumed to be periodic as $x_{k}=x_{(M+k)}$ and $y_{k}=y_{(M+k)}$. The cross-correlation function is calculated for each data block and averaged with the values from the previous data blocks. Using the cross-correlation function, the one-sided CPSD is calculated as:

$$
\begin{aligned}
& \operatorname{CPSD}(\omega)=2 \int_{-\infty}^{\infty} R_{x y}(\tau) e^{-i \omega \tau} d \tau \\
& =2 \int_{0}^{\infty} R_{x y}(\tau) e^{-i \omega \tau} d \tau+2 \int_{0}^{\infty} R_{y x}(\tau) e^{i \omega \tau} d \tau,
\end{aligned}
$$

where $0<\omega \leq \infty$ (Uhrig, 1970). The real and imaginary parts of the CPSD, respectively, in discretized form are

$$
\begin{gathered}
\operatorname{Re}\left[\operatorname{CPSD}\left(\omega_{k}\right)\right]=2\left[\sum_{n=0}^{M-1} R_{x y}(n) \cos \left(\omega_{k} n \Delta\right)+\sum_{n=0}^{M-1} R_{y x}(n) \cos \left(\omega_{k} n \Delta\right)\right], \\
\operatorname{Im}\left[\operatorname{CPSD}\left(\omega_{k}\right)\right]=2\left[\sum_{n=0}^{M-1} R_{y x}(n) \sin \left(\omega_{k} n \Delta\right)-\sum_{n=0}^{M-1} R_{x y}(n) \sin \left(\omega_{k} n \Delta\right)\right],
\end{gathered}
$$

where $\omega_{k}=2 \pi k /(\Delta \cdot M), k=0,1, \ldots, M / 2, \operatorname{Re}[]=$ real part, and $\operatorname{Im}[$ ] = imaginary part.

\subsection{Calculation results}

The theoretical values of $\operatorname{Re}\left[G_{13}(\omega)\right], \quad \operatorname{Im}\left[G_{13}(\omega)\right], \quad \operatorname{Re}\left[G_{23}(\omega)\right], \quad \operatorname{Im}\left[G_{23}(\omega)\right]$, $\operatorname{Re}[R(\omega)]$, and $\operatorname{Im}[R(\omega)]$ are compared with the results of the Monte Carlo simulation in Figs. 2, 3, 4, 5, 6, and 7, respectively. The theoretical values in these figures are in good agreement with the results of the Monte Carlo simulation. The agreement verifies the theoretical formula for the CSDNA method. The theoretical value of $R(\omega)$ at $\omega=0 \mathrm{~Hz}$ is 0.1203. On the other hand, if the diffusion approximation defined by Eqs. (35)-(37) is adopted for all modes, $R(\omega)=0.1246$ at $\omega=0 \mathrm{~Hz}$. The diffusion approximation is not sufficient to obtain quantitatively accurate results even for a simple problem considered in this example. The CPSD, $G_{23}(\omega)$, is divided into two components, $G_{23}^{f}(\omega)$ and $G_{23}^{s}(\omega)$, that are due to 
multiple emissions of induced fission neutrons and spontaneous fission neutrons, respectively. $G_{23}^{f}(\omega)$ and $G_{23}^{s}(\omega)$ are defined based on Eq. (19) as follows:

$$
G_{23}^{f}(\omega)=\sum_{m=0}^{\infty} \sum_{n=0}^{\infty}\left(\frac{B_{m 2, n 3}}{\alpha_{n}+i \omega}+\frac{B_{m 3, n 2}}{\alpha_{n}-i \omega}\right),
$$

where

$$
\begin{aligned}
B_{m 2, n 3} & =\sum_{\ell=0}^{\infty}\left(\frac{S_{\ell} F_{\ell \rightarrow m n}}{\alpha_{\ell}}\right) \frac{D_{2 m} D_{3 n}}{\left(\alpha_{m}+\alpha_{n}\right)}, \\
G_{23}^{s}(\omega) & =\sum_{m=0}^{\infty} \sum_{n=0}^{\infty} \frac{S_{m n}}{\alpha_{m}+\alpha_{n}}\left(\frac{D_{2 m} D_{3 n}}{\alpha_{n}+i \omega}+\frac{D_{3 m} D_{2 n}}{\alpha_{n}-i \omega}\right) .
\end{aligned}
$$

To see the contribution of each component, Fig. 8 shows the theoretical values of the real parts of $G_{23}(\omega), G_{23}^{f}(\omega)$, and $G_{23}^{s}(\omega)$. Fig. 8 illustrates merely one example of $G_{23}^{f}(\omega)$ and $G_{23}^{S}(\omega)$. The contribution of two components depends on the locations of ${ }^{252} \mathrm{Cf}$ source and two detectors and many other factors.

[Fig. 2],[Fig. 3],[Fig. 4],[Fig. 5],[Fig. 6],[Fig. 7],[Fig. 8]

If the maximum order of the higher harmonics is not large enough, the theoretical value of $R(\omega)$ depends on the maximum order of the higher harmonics that was considered. Fig. 9 shows the theoretical values of $R(\omega)$ at $\omega=0 \mathrm{~Hz}(=R(0))$ as a function of the number of the maximum order considered (shown as “Case 1"). This example exhibits a relatively modest variation of $R(0)$ with the number of the maximum order. "Case 2" in Fig. 9 is another example in which detectors 1, 2, and 3 are located at $x=41.4 \mathrm{~cm}, 27.5 \mathrm{~cm}$ (center of the slab), and $13.6 \mathrm{~cm}$, respectively. In Case 2, the fundamental mode approximation largely overestimates $R(0)$ compared with the fully converged one.

[Fig. 9]

Table 2 shows how $R(0)$ depends on the arrangement of the three detectors. The values of $k_{\text {eff }}$ in Table 2 were estimated by substituting $R(0)$ in Table 2 into $R_{0}$ in Eq. (30). The estimated $k_{\text {eff }}$ in Table 2 ranges from 0.954 to 0.976 . To investigate the effect of subcriticality on the estimated $k_{\text {eff, }}$ consider a nearly critical system $\left(k_{\text {eff }}=0.99854\right)$ as another numerical example where $\Sigma_{f}=0.0531 \mathrm{~cm}^{-1}$, but the geometry and other constants are the same as in the previous example. For this nearly critical system, all eigenvalues and eigenfunctions are 
obtained by diffusion theory. The $\alpha$-eigenvalues up to the third higher harmonic are shown in Table 3. Table 4 shows calculation results for the nearly critical system. The arrangements of the detectors on which Table 4 is based are the same as for Table 2. The spectral ratios, $R(0)$, are close to the ratios by the fundamental mode approximation, $R_{0}$, regardless of the arrangement of detectors 2 and 3 because $\alpha_{0}<<\alpha_{1}<\alpha_{2}<\cdots$. The estimated $k_{\text {eff' }}$ s agree with the exact one to four decimal places. As $k_{\text {eff }}$ decreases, the range of variation becomes wider as can be seen in Tables 2 and 4. On the other hand, in a nearly critical system (Table 4), the estimated $k_{\text {eff }}$ is expected to be independent of the arrangement of the three detectors.

[Table 2], [Table 3], [Table 4]

\section{Another Method for Inferring $k_{\text {eff }}$}

As shown in Sec. 3, the ratio of spectral densities significantly varies with the locations of the detectors except in a nearly critical system. The ratio of spectral densities, $R(0)$, that takes into account a sufficient number of higher harmonics differs from $R_{0}$ obtained by the fundamental mode approximation. Thus, using $R(0)$ in Eq. (30) instead of $R_{0}$ is likely to result in ambiguity as to estimating $k_{\text {eff }}$. Another method for inferring $k_{\text {eff }}$ using the ratio of spectral densities was proposed by Mihalczo et al. (1997) and Valentine (1999). In that method, a Monte Carlo simulation and a measurement are performed for a subcritical system to which the CSDNA method is applied to obtain the ratios of spectral densities. The ratios of spectral densities obtained by the Monte Carlo simulation and the measurement are denoted by $R_{c}$ and $R_{m}$, respectively. The bias in the ratio of spectral densities is defined as $R_{m}-R_{c}$; it is attributed to the insufficient representation of the nuclear data and the Monte Carlo model. In addition to the Monte Carlo simulation for the CSDNA method, a criticality calculation is performed to obtain $k_{e f f}$, which is denoted by $k_{c}$. The same Monte Carlo code and nuclear data are used for both the simulation of the CSDNA method and the $k_{\text {eff }}$ calculation. It is assumed that if $R_{m}=R_{c}$, there is no bias in the criticality calculation, and $k_{c}$ is equal to the 


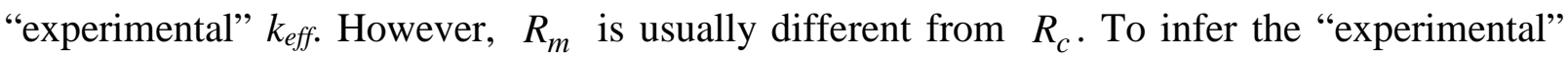
$k_{e f f}$, the Monte Carlo model is slightly perturbed. A Monte Carlo simulation for the CSDNA method and a criticality calculation are performed for the perturbed system. The “experimental” $k_{\text {eff }}$ is determined from the following relationship:

$$
\frac{R_{m}-R_{c}}{k_{m}-k_{c}}=\frac{R_{p}-R_{c}}{k_{p}-k_{c}},
$$

where $k_{m}=$ the "experimental" $k_{e f f}, R_{p}=$ the ratio of spectral density in the perturbed system, and $k_{p}=k_{\text {eff }}$ in the perturbed system. Eq. (46) assumes that the sensitivity of the ratio of spectral densities to $k_{\text {eff }}$ due to the bias in the nuclear data and the Monte Carlo model is the same as that due to the intentionally added perturbation. Eq. (46) also assumes a linear relationship between the ratio of spectral densities and $k_{\text {eff. }}$ Thus, the perturbation must be sufficiently small that the linearity holds. Furthermore, the perturbation must be large enough such that it is greater than the uncertainties in the simulated parameters. If the "experimental" $k_{\text {eff }}$ obtained from Eq. (46) depends on the type of perturbation, the "experimental” $k_{\text {eff }}$ is not a uniquely determined value and is not truly “experimental”. In the point-kinetics approximation in Eq. (34), the "experimental” $k_{\text {eff }}$ is completely independent of the type of perturbation unless $v$ is changed. Perez et al. (1997) demonstrated that Eq. (46) is valid using first-order perturbations of the transport operators for the ratio of spectral densities and $k_{\text {eff. }}$ Because the ratio of spectral densities is given by the involved formula in Eq. (23), the uniqueness of $\left(R_{p}-R_{c}\right) /\left(k_{p}-k_{c}\right)$ is not always straightforwardly explained. Valentine et al. (2000) applied Eq. (46) to inferring the "experimental” $k_{\text {eff }}$ of a highly enriched uranyl nitrate solution contained in a cylindrical tank. Perturbations were made to the uranium density, the solution density, the tank dimensions, and the uranium enrichment. The sensitivities, $\left(R_{p}-R_{c}\right) /\left(k_{p}-k_{c}\right)$, were calculated from the data in (Valentine et al., 2000) and are shown in Tables 5 and 6 . All calculated data $\left(R_{p}, R_{c}, k_{p}\right.$, and $\left.k_{c}\right)$ that are needed for Tables 5 and 6 were calculated by the authors of (Valentine et al., 2000) with MCNP-DSP code (Valentine and 
Mihalczo, 1996), a modified version of MCNP for simulating the CSDNA method. The sensitivity in Table 5 seems to be relatively independent of the type of perturbation. On the other hand, the sensitivity in Table 6 , where $k_{\text {eff }}$ is much lower, is more sensitive to the type of perturbation. This finding is accentuated in another subcritical experiment in (Blakeman et al., 2008). This experiment was performed for configurations of two coaxial, cylindrical tanks of highly-enriched uranyl nitrate solution. While the sensitivities, $\left(R_{p}-R_{c}\right) /\left(k_{p}-k_{c}\right)$, ranged from -0.98 to -1.06 for $k_{e f f} \sim 0.94$, they did from -0.06 to -1.42 for $k_{\text {eff }} \sim 0.86$. It is presumed that how independent the sensitivity, $\left(R_{p}-R_{c}\right) /\left(k_{p}-k_{c}\right)$, is of the type of perturbation depends on its subcriticality. Our discussion does not accurately explain the uniqueness of the “experimental” $k_{\text {eff }}$ inferred by Eq. (46). However, the variation of the inferred "experimental” $k_{\text {eff }}$ is eventually very minor regardless of the type of perturbation. This method was applied to a plutonium metal system (Valentine, 2003), a highly enriched metal system (Blakeman et al., 2006). The review in these evaluation reports in the International Handbook of Evaluated Criticality Safety Benchmark Experiments (ICSBEP) claims that the method using Eq. (46) may be a practically useful method to infer "experimental” $k_{\text {eff }}$ with an acceptable level of accuracy.

[Table 5], [Table 6]

\section{Conclusions}

A theoretical formula for the ratio of spectral densities that is measured in the CSDNA method is derived in this paper. Although similar formulations were derived in previous papers, no numerical example or verification has been presented. The present paper has shown that numerical tests in the one-dimensional infinite slab verify the formula for the ratio. The forward and adjoint kinetic mode ( $\alpha$-mode) eigenvalues and eigenfunctions that are included in the formula were obtained by solving the $\alpha$-mode transport equation up to the third higher harmonic. Beyond the fourth higher harmonic, the diffusion approximation was applied to obtain the eigenvalues and the eigenfunctions. The theoretical values reproduce well the results 
calculated by the Monte Carlo simulation for the CSDNA method. The theoretical formula is deemed to be verified through the comparison with the Monte Carlo simulation.

By neglecting higher harmonics except for the fundamental mode, the ratio of spectral densities can be related to $k_{e f f}$ or $\rho\left(=1-1 / k_{e f f}\right)$ as seen in Eq. (30). However, a measured ratio of spectral densities is contaminated by the higher harmonics unless the system is nearly critical. Using a measured ratio of spectral densities in Eq. (30) does not yield a unique $k_{\text {eff }}$ because it depends significantly on the locations of the detectors in the CSDNA method.

An alternative method for inferring a unique $k_{\text {eff }}$ using the CSDNA method is based on the assumption that the sensitivity of the spectral ratio to the $k_{\text {eff }}$ is independent of the type of perturbation. This assumption is valid for a nearly critical system where the fundamental mode is dominant over the remaining higher harmonics. This paper does not succeed in showing the theoretical justification of the assumption, and the sensitivity is not completely independent of the type of perturbation. However, according to the review of the previous results in the ICSBEP evaluation reports, the proposed method can be a practically available subcriticality determination technique because an inferred "experimental" $k_{\text {eff }}$ can be almost uniquely determined regardless of the type of perturbation.

\section{References}

Akcasu, A. Z., Stolle, A.M., 1993. Langevin equation approach to reactor noise analysis: stochastic transport equation, Nucl. Sci. Eng., 113, 31-55.

Blakeman, E.D., et al., 2006. Water moderated U(93.15 wt.\%) aluminum research reactor fuel element subcritical noise measurements. SUB-HEU-MET-THERM-001, International Handbook of Evaluated Criticality Safety Benchmark Experiments, NEA/NSC/DOC(95)03, Nuclear Energy Agency, Organisation for Economic Co-operation and Development.

Blakeman, E.D., et al., 2008. Subcritical noise measurements for two coaxial cylindrical tanks containing 93.1\% uranyl nitrate solution. SUB-HEU-SOL-THERM-002, International 
Handbook of Evaluated Criticality Safety Benchmark Experiments, NEA/NSC/DOC(95)03, Nuclear Energy Agency, Organisation for Economic Co-operation and Development.

Difilippo, F.C., 1990. Harmonic analysis of stochastic descriptors and the interpretation of ${ }^{252}$ Cf neutron source experiments. Nucl. Sci. Eng., 104, 123-134.

Endo, T., Yamane, Y., Yamamoto, A., 2006. Space and energy dependent theoretical formula for the third order neutron correlation technique. Ann. Nucl. Energy, 33, 521-537.

Ficaro, E.P., Wehe, D.K., 1994. KENO-NR: a Monte Carlo code for simulating ${ }^{252}$ Cf-source driven noise measurements to determine subcriticality. Nucl. Sci. Eng., 117, 158-176.

Hutchinson, J., Valentine, T., 2009. Subcritical measurements of a Plutonium sphere reflected by polyethylene and acrylic. Nucl. Sci. Eng., 161, 357-362.

Hutchinson, J.D., Bess, J.D., 2009. Subcritical noise measurements with a Nickel-reflected Plutonium sphere. Nucl. Sci. Eng., 163, 285-290.

Mihalczo, J.T., Paré, V.K., Ragan, G.L., Mathis, M.V., Tillett, G.C., 1978. Determination of reactivity from power spectral density measurements with Californium-252. Nucl. Sci., Eng., 66, 29-59.

Mihalczo, J.T., Blakeman, E.D., Ragan, G.E., Johnson, E.B., Hachiya, Y., 1990. Dynamic subcriticality measurements using the ${ }^{252}$ Cf-source-driven noise analysis method. Nucl. Sci. Eng., 104, 314-338.

Mihalczo, J.T., Blakeman, E.D., Ragan, G.E., Kryter, R.C, Seino, H., Robinson, R.C, 1991. ${ }^{252}$ Cf-source-driven noise measurements of subcriticality for a slab tank of aqueous Pu-U nitrate. Nucl. Sci. Eng., 107, 35-57.

Mihalczo, J.T., Valentine, T.E., 1995. Calculational verification and process control applications utilizing the high sensitivity of noise measurement parameters to fissile system configuration. Nucl. Sci. Eng., 121, 286-300.

Mihalczo, J.T., Valentine, T.E., Phillips, L.D., 1997. Subcritical measurements for a HEU storage vault at the Y-12 plant using the ${ }^{252} \mathrm{Cf}$-source-driven frequency-analysis method. 
Nucl. Safety, 38, 10-24.

Paré, V.K., Mihalczo, J.T., 1975. Reactivity from power spectral density measurements with Californium-252. Nucl. Sci. Eng., 57, 213-218.

Perez, R.B., Valentine, T.E., Mihalczo, J.T., Mattingly, J.K., (1997). Determination of the multiplication factor and its bias by the ${ }^{252} \mathrm{Cf}$-source technique: a method for code benchmarking at subcritical configurations. Proc. Joint Int. Conf. Mathematical Methods and Supercomputing for Nuclear Applications, Saratoga, New York, October 5-7, 1997, American Nuclear Society.

Spriggs, G.D, Busch, R.D, Sakurai, T., Okajima, S., 1999. The equivalent fundamental-mode source. Ann. Nucl. Energy, 26, 237-264.

Sutton, T.M., Doub, W.B., 1991. Stochastic transport theory analysis of the ${ }^{252} \mathrm{Cf}$ source driven noise technique. Ann. Nucl. Energy, 18, 491-502.

Valentine, T.E., Mihalczo, J.T., 1996. MCNP-DSP: a neutron and gamma ray Monte Carlo calculation of source-driven noise-measured parameters. Ann. Nucl. Energy, 23, 1271-1287.

Valentine, T.E., Mihalczo, J.T., 1997. Validation of the Monte Carlo code MCNP-DSP. Ann. Nucl. Energy, 24, 78-98.

Valentine, T.E., 1999. Review of subcritical source-driven noise analysis measurements. ORNL/TM-1999/28.

Valentine, T.E., 2000. Unreflected high-enriched uranyl nitrate subcritical noise measurements. SUB-HEU-SOL-THERM-001, International Handbook of Evaluated Criticality Safety Benchmark Experiments, NEA/NSC/DOC(95)03, Nuclear Energy Agency, Organisation for Economic Co-operation and Development.

Valentine, T.E., 2003. Polyethylene-reflected Plutonium metal sphere subcritical noise measurements. SUB-PU-MET-FAST-001, International Handbook of Evaluated Criticality Safety Benchmark Experiments, NEA/NSC/DOC(95)03, Nuclear Energy Agency, Organisation for Economic Co-operation and Development. 
Uhrig, R.E., 1970. Random noise techniques in nuclear reactor systems. The Ronald Press Company, New York.

Yamane, Y., Nishina, K., 1986. Subcriticality determination of nuclear fuel assembly by Mihalczo method. J. At. Energy Soc. Jpn, 28, 850-858 [in Japanese].

Yamane, Y., Yanagisawa, H., Nishina, K., 1987. Derivation of space-dependent formula for the Cf-252 source-driven neutron noise experiment. Proc. Int. Seminar on Nucl. Criticality Safety, ISCS’87, Tokyo, Japan, Oct. 19-23, 1987, 393-400.

Yamamoto, T., 2011a. Higher order mode analyses in Feynman- $\alpha$ method. Ann. Nucl. Energy, $38,1231-1237$.

Yamamoto, T., 2011b. Higher order $\alpha$ mode eigenvalue calculation by Monte Carlo power iteration. Progress in Nuclear Science and Technology, 2, 826-835.

Yamamoto, T., 2014a, Frequency domain Monte Carlo simulation method for cross power spectral density driven by periodically pulsed neutron source using complex-valued weight Monte Carlo. Ann. Nucl. Energy, 64, 711-720.

Yamamoto, T., 2014b. Higher order mode analyses of power spectral density and Feynman- $\alpha$ method in accelerator driven system with periodically pulsed spallation neutron source. Ann. Nucl. Energy, 66, 63-73. 


\section{Figure captions}

Figure 1. Detector and source arrangements in the one-dimensional infinite slab for test calculations.

Figure 2. Comparison of $\operatorname{Re}\left[G_{13}(\omega)\right]$ between the theory and the Monte Carlo simulation.

Figure 3. Comparison of $\operatorname{Im}\left[G_{13}(\omega)\right]$ between the theory and the Monte Carlo simulation.

Figure 4. Comparison of $\operatorname{Re}\left[G_{23}(\omega)\right]$ between the theory and the Monte Carlo simulation.

Figure 5. Comparison of $\operatorname{Im}\left[G_{23}(\omega)\right]$ between the theory and the Monte Carlo simulation.

Figure 6. Comparison of $\operatorname{Re}[R(\omega)]$ between the theory and the Monte Carlo simulation.

Figure 7. Comparison of $\operatorname{Im}[R(\omega)]$ between the theory and the Monte Carlo simulation.

Figure 8. Contributions of $\operatorname{Re}\left[G_{23}^{f}(\omega)\right]$ and $\operatorname{Re}\left[G_{23}^{s}(\omega)\right]$ in $\operatorname{Re}\left[G_{23}(\omega)\right]$.

Figure 9. Variations of $R(\omega)$ at $\omega=0 \mathrm{~Hz}(=R(0))$ with the number of maximum order (the mode order "1" corresponds to the fundamental mode). 


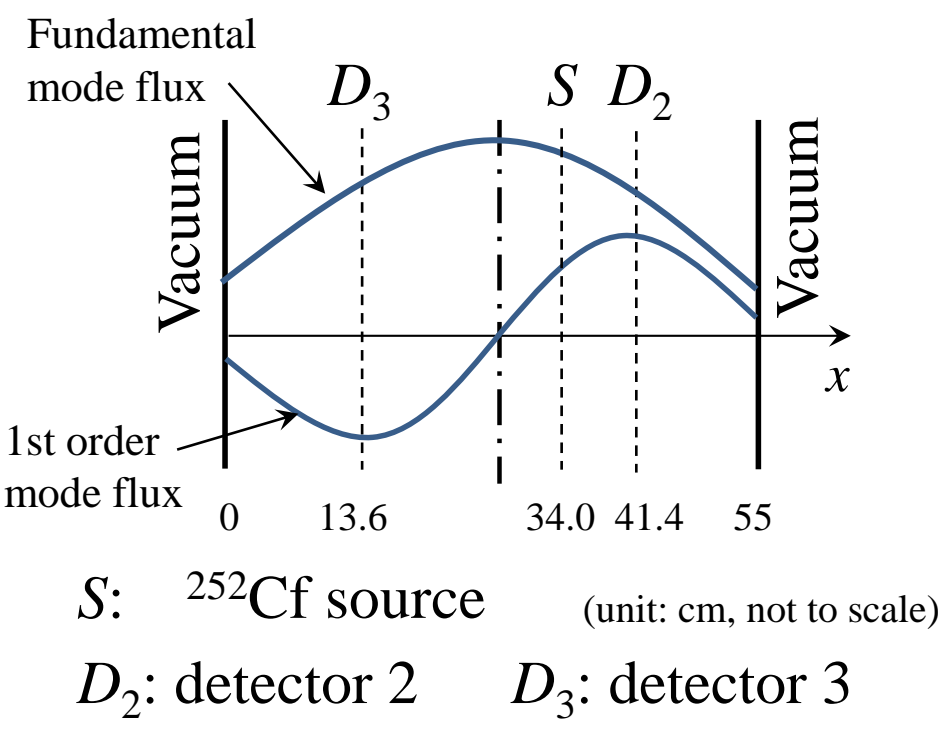

Fig. 1 


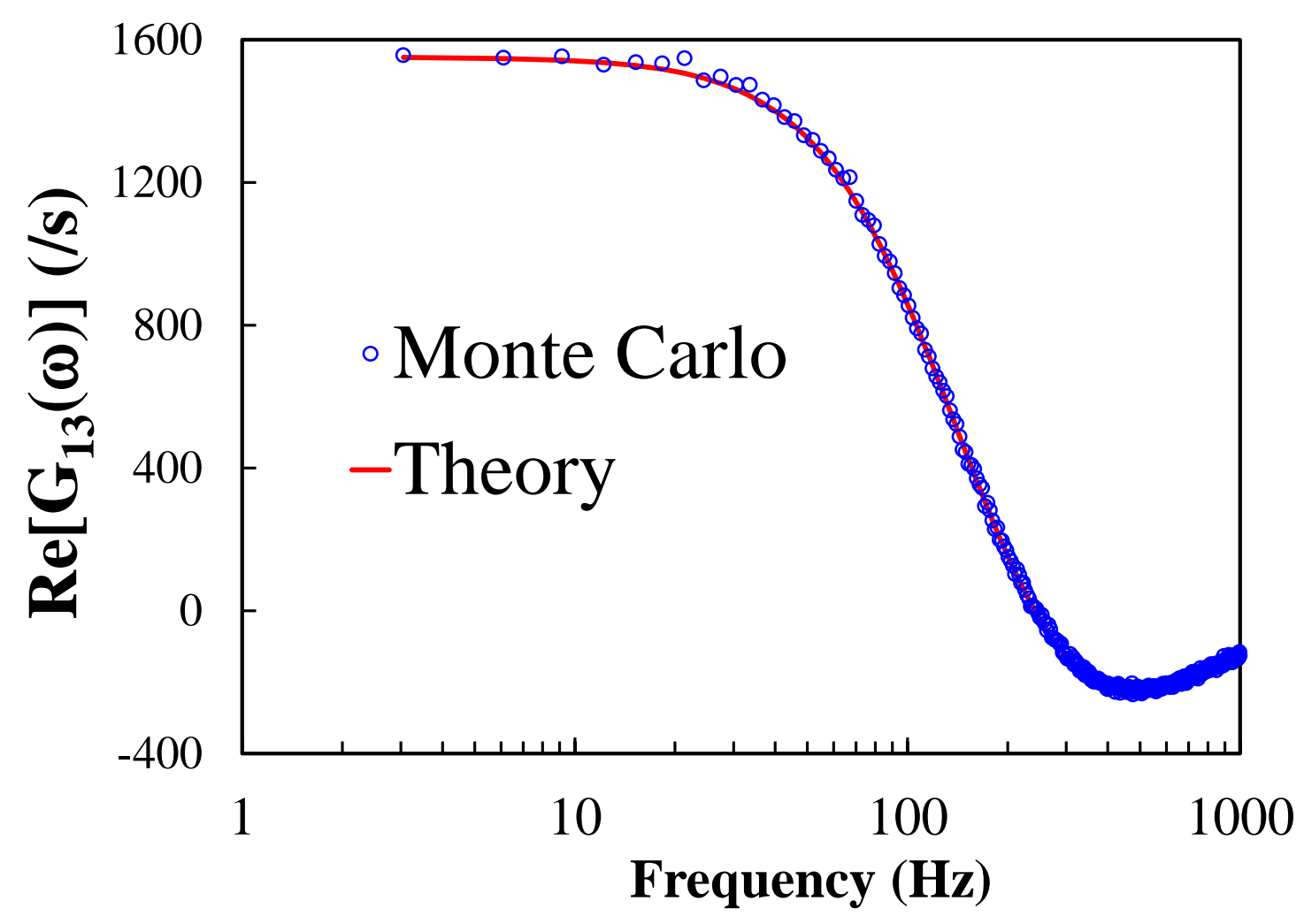

Fig. 2 


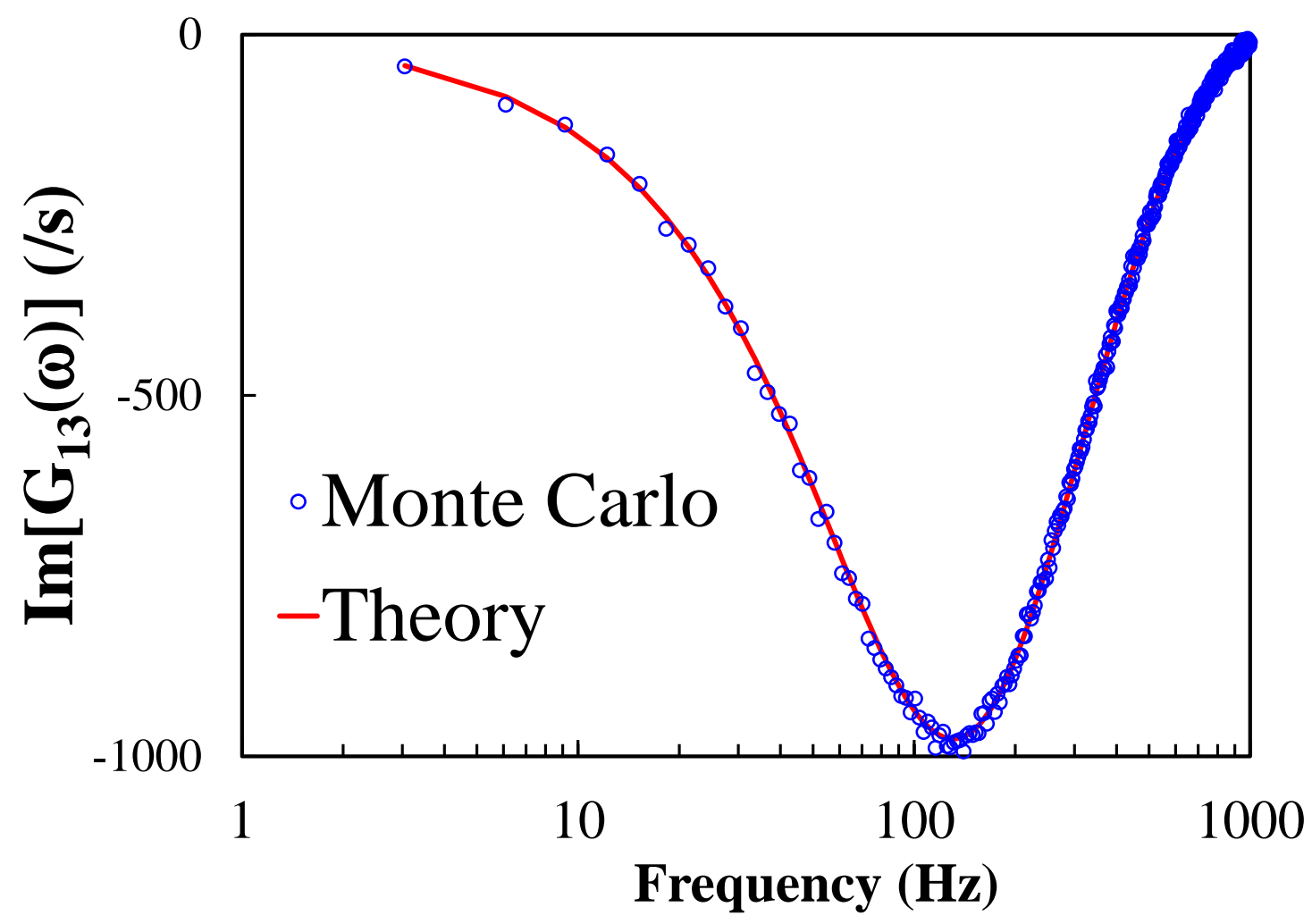

Fig. 3 


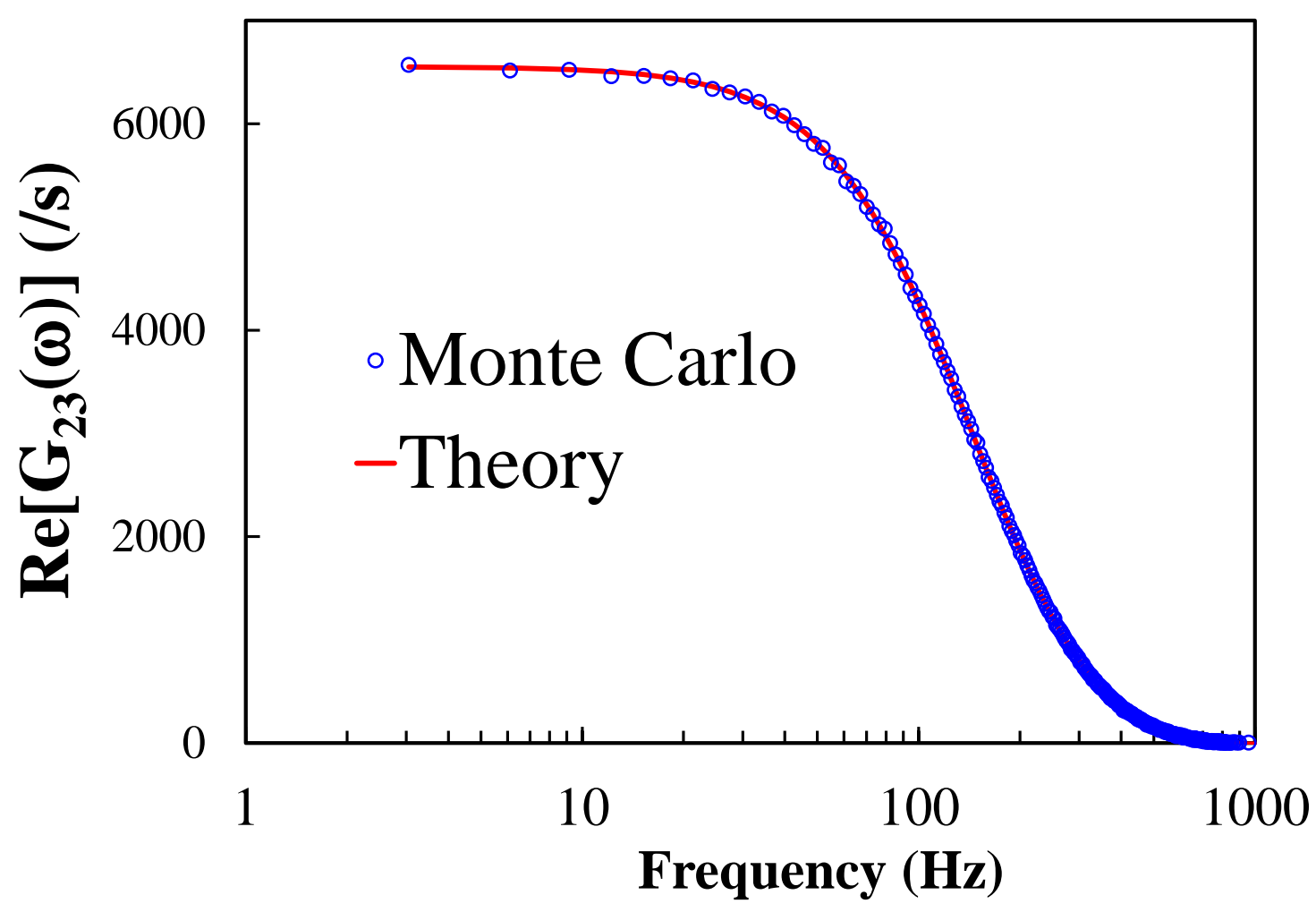

Fig. 4 


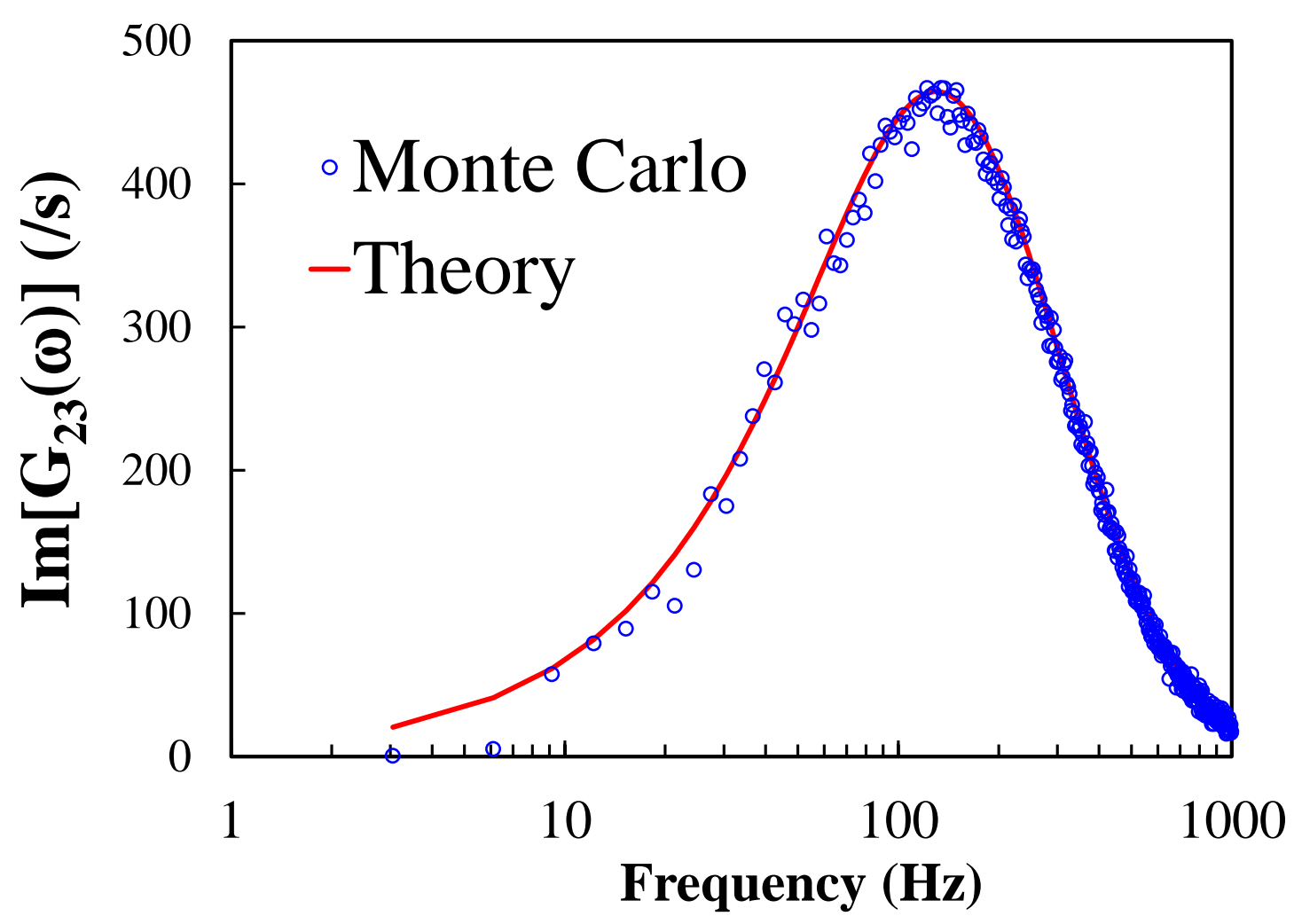

Fig. 5 


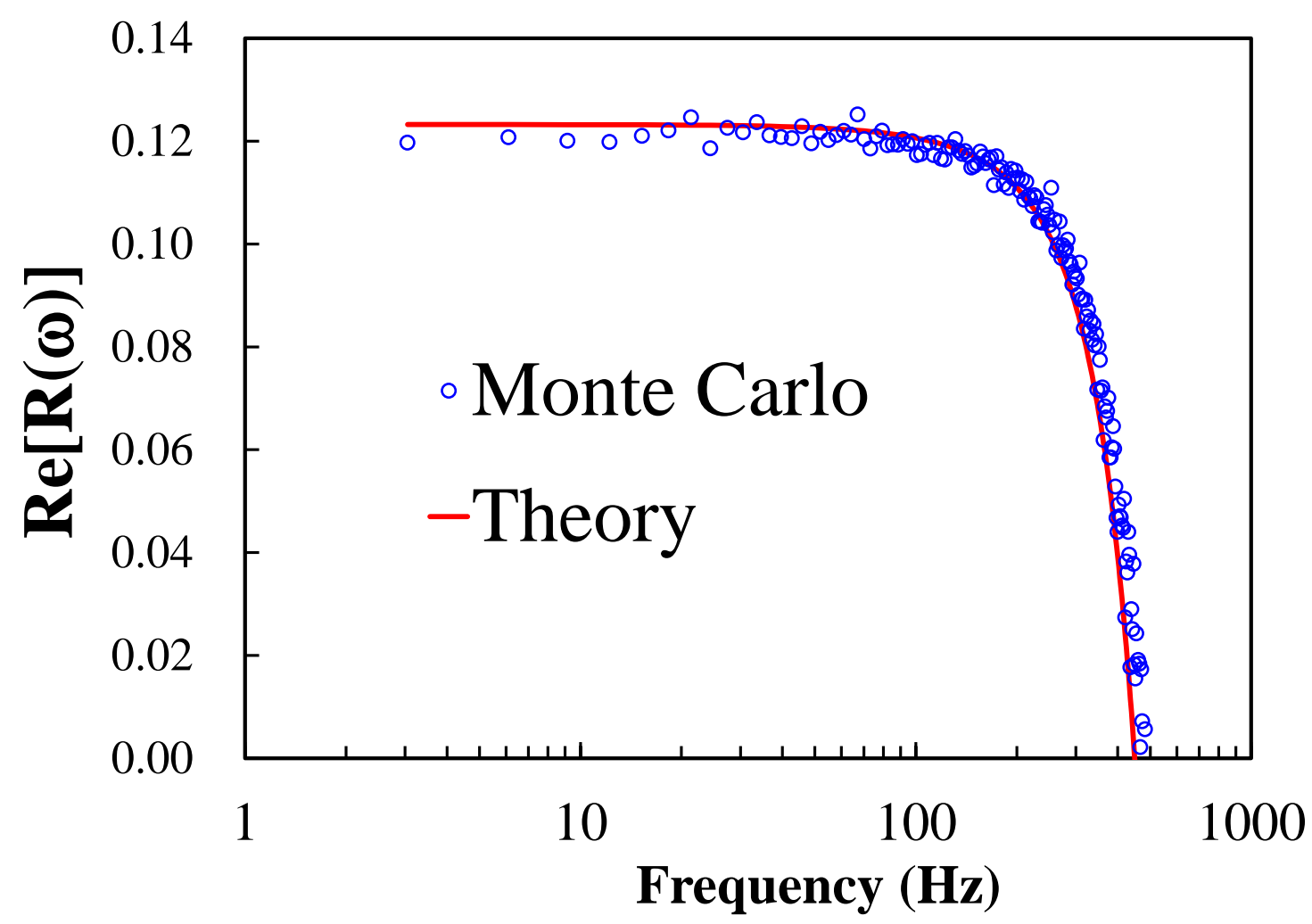

Fig. 6 


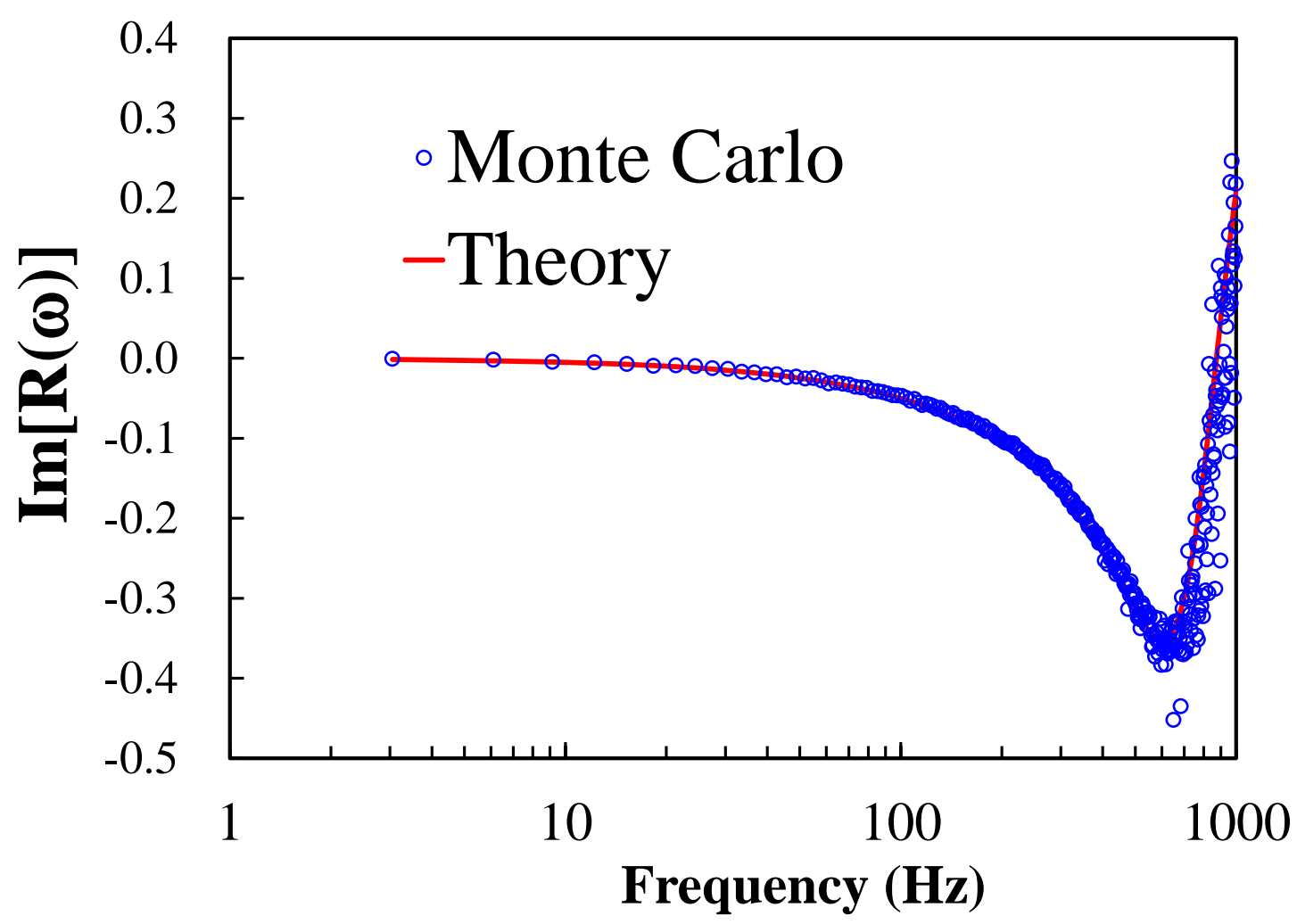

Fig. 7 


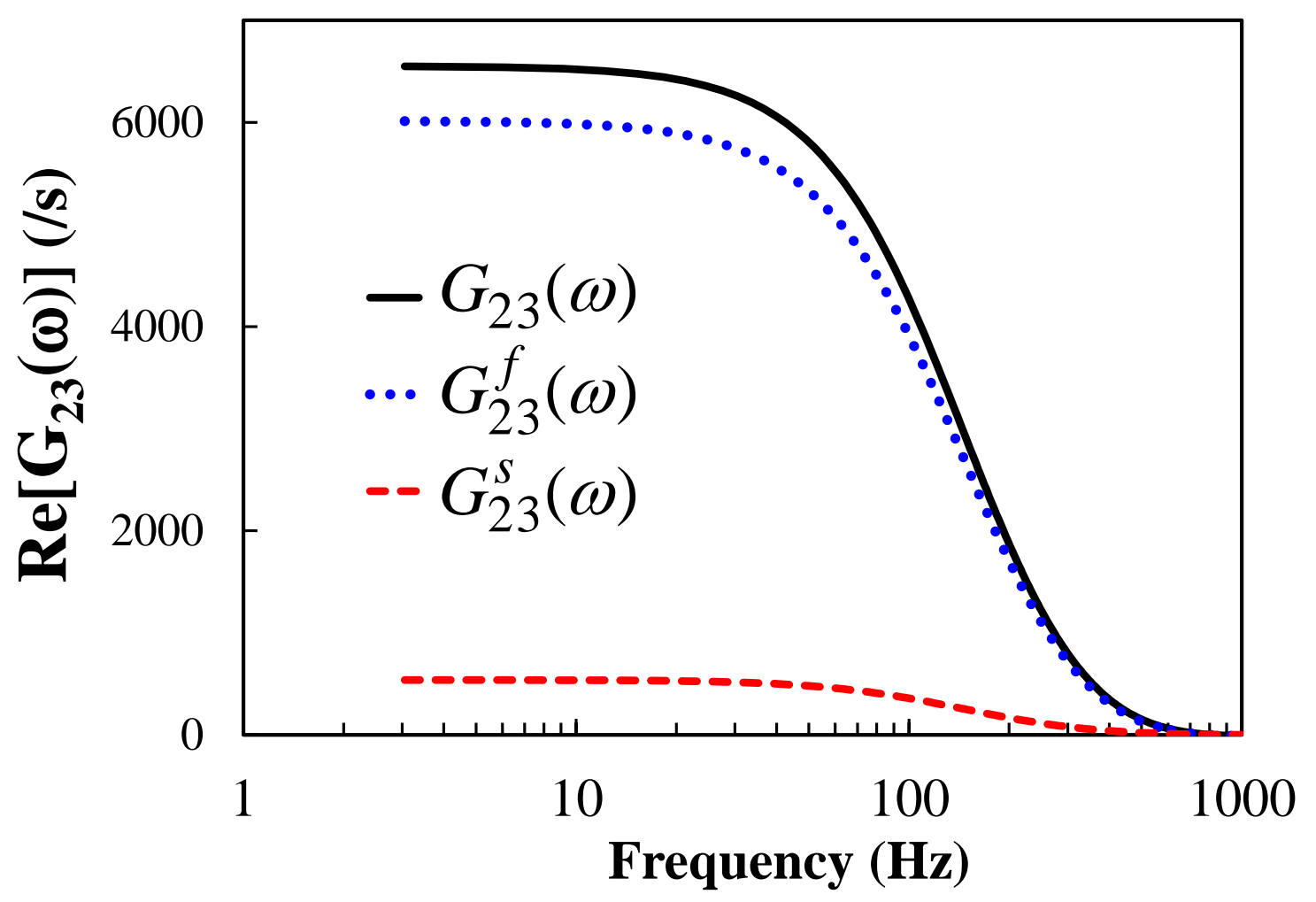

Fig. 8 


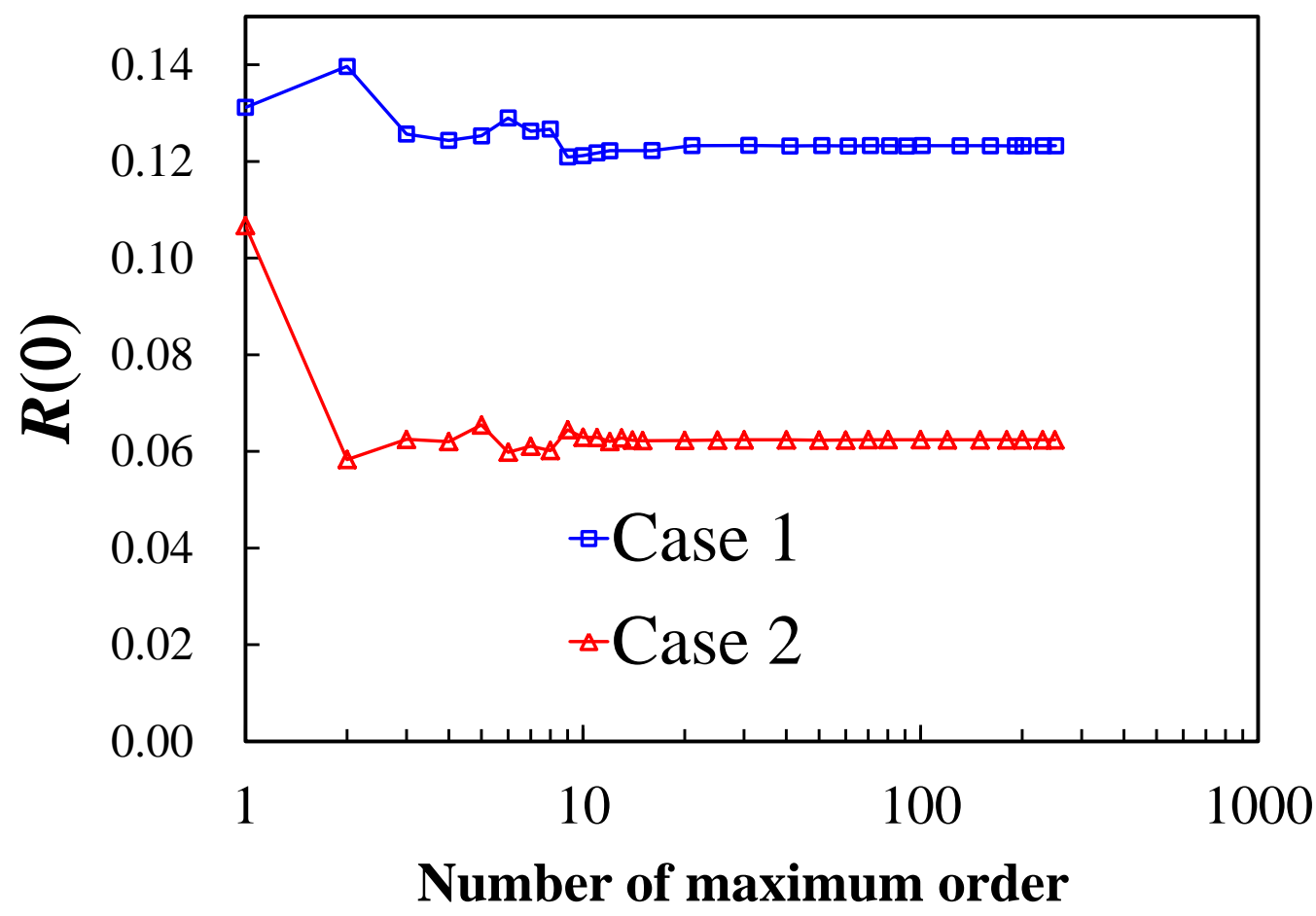


Table 1 Comparison of $k_{\text {eff }}$ and $\alpha_{n}$ between the diffusion theory and the Monte Carlo calculations

\begin{tabular}{lcc}
\hline & Diffusion theory & Monte Carlo \\
\hline$k_{\text {eff }}$ & 0.95838 & $0.95865 \pm 0.00002^{\mathrm{a}}$ \\
$\alpha_{0}\left(\mathrm{~s}^{-1}\right)$ & 936.25 & $939.94 \pm 0.50$ \\
$\alpha_{1}\left(\mathrm{~s}^{-1}\right)$ & 3085.0 & $3120.8 \pm 0.6$ \\
$\alpha_{2}\left(\mathrm{~s}^{-1}\right)$ & 6666.3 & $6825.2 \pm 0.8$ \\
$\alpha_{3}\left(\mathrm{~s}^{-1}\right)$ & 11680 & $12166 \pm 2$ \\
\hline${ }^{\mathrm{a}}$ one standard deviation &
\end{tabular}


Table 2 Spectral ratios at $\omega=0 \mathrm{~Hz}$ and estimated $k_{\text {eff }}$ for several detector arrangements.

\begin{tabular}{cccccc}
\hline $\begin{array}{c}\text { Detector 1 } \\
\left({ }^{252} \mathrm{Cf}\right)\end{array}$ & Detector 2 & Detector 3 & $R(0)$ & $k_{\text {eff }}{ }^{\mathrm{a}}$ & $R_{0}{ }^{\mathrm{b}}$ \\
\hline $\mathrm{A}$ & $\mathrm{B}$ & $\mathrm{C}$ & 0.1203 & 0.9622 & \\
$\mathrm{~A}$ & $\mathrm{~B}$ & $\mathrm{D}$ & 0.1464 & 0.9535 & 0.1312 \\
$\mathrm{~A}$ & $\mathrm{D}$ & $\mathrm{C}$ & 0.0993 & 0.9690 & \\
\hline $\mathrm{B}$ & $\mathrm{D}$ & $\mathrm{C}$ & 0.0614 & 0.9763 & \\
B & $\mathrm{A}$ & $\mathrm{C}$ & 0.0837 & 0.9675 & 0.1057 \\
$\mathrm{~B}$ & $\mathrm{~A}$ & $\mathrm{D}$ & 0.0981 & 0.9617 & \\
\hline
\end{tabular}

A: $x=34.0 \mathrm{~cm}, \mathrm{~B}: x=41.4 \mathrm{~cm}, \mathrm{C}: x=13.6 \mathrm{~cm}, \mathrm{D}: x=27.5 \mathrm{~cm}$ (middle point of the slab)

a The exact $k_{\text {eff }}$ is 0.9587 .

${ }^{\mathrm{b}}$ Fundamental mode approximation 
Table $3 \alpha_{n}$ in the nearly critical system

\begin{tabular}{cc}
\hline$n$ & $\alpha_{n}\left(s^{-1}\right)$ \\
\hline 0 & 34.25 \\
1 & 2183 \\
2 & 5764 \\
3 & 10778 \\
\hline
\end{tabular}


Table 4 Spectral ratios at $\omega=0 \mathrm{~Hz}$ and estimated $k_{\text {eff }}$ for several detector arrangements in the nearly critical system

\begin{tabular}{cccccc}
\hline $\begin{array}{c}\text { Detector } 1 \\
\left({ }^{252} \mathrm{Cf}\right)\end{array}$ & Detector 2 & Detector 3 & $R(0)$ & $k_{\text {eff }}{ }^{\mathrm{a}}$ & $R_{0}{ }^{\mathrm{b}}$ \\
\hline $\mathrm{A}$ & $\mathrm{B}$ & $\mathrm{C}$ & 0.004847 & 0.99854 & \\
$\mathrm{~A}$ & $\mathrm{~B}$ & $\mathrm{D}$ & 0.004922 & 0.99852 & 0.004872 \\
$\mathrm{~A}$ & $\mathrm{D}$ & $\mathrm{C}$ & 0.004797 & 0.99856 & \\
\hline $\mathrm{B}$ & $\mathrm{D}$ & $\mathrm{C}$ & 0.003745 & 0.99858 & \\
$\mathrm{~B}$ & $\mathrm{~A}$ & $\mathrm{C}$ & 0.003806 & 0.99855 & 0.003855 \\
$\mathrm{~B}$ & $\mathrm{~A}$ & $\mathrm{D}$ & 0.003848 & 0.99854 & \\
\hline
\end{tabular}

A: $x=34.0 \mathrm{~cm}, \mathrm{~B}: x=41.4 \mathrm{~cm}, \mathrm{C}: x=13.6 \mathrm{~cm}, \mathrm{D}: x=27.5 \mathrm{~cm}$ (middle point of the slab)

${ }^{\mathrm{a}}$ The exact $k_{\text {eff }}$ is 0.99854 .

${ }^{\mathrm{b}}$ Fundamental mode approximation 
Table 5 Sensitivities and "experimental" $k_{\text {eff }}$ for Case 1 in SUB-HEU-SOL-THERM-001 (solution height: $30.48 \mathrm{~cm}, k_{c}=0.9599$ ).

\begin{tabular}{l|c|c}
\hline & $\left(R_{p}-R_{c}\right) /\left(k_{p}-k_{c}\right)$ & "Experimental" $k_{\text {eff }}$ \\
\hline Solution density $+2 \%$ & -2.40 & 0.9630 \\
Uranium density $-3 \%$ & -2.42 & 0.9630 \\
Uranium density $+3 \%$ & -2.31 & 0.9632 \\
Enrichment $93.2 \% \rightarrow 80 \%$ & -2.24 & 0.9632 \\
Radius $+1.1 \%$ & -2.34 & 0.9631 \\
\hline
\end{tabular}


Table 6 Sensitivities and "experimental" $k_{\text {eff }}$ for Case 4 in SUB-HEU-SOL-THERM-001 (solution height: $22.86 \mathrm{~cm}, k_{c}=0.8829$ ).

\begin{tabular}{l|c|c}
\hline & $\left(R_{p}-R_{c}\right) /\left(k_{p}-k_{c}\right)$ & "Experimental" $k_{\text {eff }}$ \\
\hline Solution density $+2 \%$ & -1.74 & 0.8895 \\
Uranium density $-3 \%$ & -1.73 & 0.8895 \\
Uranium density $+3 \%$ & -1.93 & 0.8889 \\
Enrichment $93.2 \% \rightarrow 80 \%$ & -2.09 & 0.8884 \\
Radius $+1.1 \%$ & -1.79 & 0.8893 \\
\hline
\end{tabular}

